\title{
Urinary Tract Infections in Renal Transplant Recipients
}

\author{
Justyna Gołębiewska and Alicja Dębska-Ślizień \\ Additional information is available at the end of the chapter
}

http://dx.doi.org/10.5772/intechopen.72430

\begin{abstract}
Renal transplantation (RTx) is the treatment-of-choice for a significant number of patients with end-stage renal disease. Despite recent accomplishments, both surgical and medical complications still exist. Urinary tract infection (UTI) is the most common infectious complication after RTx, while asymptomatic bacteriuria is the most common manifestation of bacteriuria. UTI can impair graft function, potentially reducing graft and patient survival. The aetiology changes with time after RTx. The epidemiology of most of these infections is also changing with resistant organisms being isolated more often than in the past. Several factors increase the risk of infection in RTx patients, and the presence of multiple risk factors in the same patient is not uncommon. These include immunosuppression, urinary flow impairment (most often caused by stenosis or strictures at the vesicoureteral junction, benign prostate hypertrophy or vesicoureteral reflux), and treatment-related factors such as the use of catheters and double-J stents. Early diagnosis and effective treatment are key elements in salvaging both the allograft and the patient. This chapter reviews the definitions, epidemiology, microbiology, screening, clinical manifestations, diagnosis, impact on renal allograft function, evaluation after diagnosis, treatment, prevention including long-term prophylaxis, and the unique challenges of diagnosing and managing recurrent bacterial UTIs in a RTx care setting.
\end{abstract}

Keywords: urinary tract infections, renal transplantation, treatment, prevention, renal allograft function

\section{Introduction}

Kidney transplantation is the renal replacement therapy of choice for the constantly increasing number of patients with end-stage renal disease (ESRD). The huge headway in immunosuppressive treatment has resulted in improved renal graft survival rates, at the same time making infectious complications an even more common problem in the 
renal transplant (RTx) population, with the urinary tract being the most prevalent infection site. Apart from immunodeficiency resulting from the use of immunosuppressive drugs, RTx patients often suffer from numerous urological malformations, vesicoureteral reflux (VUR) that is a permanent symptom after RTx, and are exposed to invasive diagnostic and therapeutic procedures involving the urinary tract. That is why urinary tract infections (UTIs) are the most common infectious complication among RTx recipients with up to $60 \%$ prevalence during the first year post-transplant [1,2]. UTIs are important not only because of the scale of the problem but due to their potential negative influence on graft and RTx recipients' outcomes.

\section{Epidemiology}

Urinary tract infections (UTIs) are major causes of morbidity and hospitalization in renal transplant recipients. Infections, with the urinary tract as a major site, are the most common cause of acute kidney allograft injury, and prevalence of UTI-associated acute kidney injury far outnumbers episodes of acute rejection and calcineurin inhibitor toxicity [3].

There is a wide variation in the reported incidence of UTIs, most likely associated with differences in the definition of UTI, length of follow-up and variation in the use of posttransplant antibiotic prophylaxis. In a recently published meta-analysis on the prevalence and predictive factors of UTI in patients undergoing renal transplantation that included 13 studies with a total of 3364 patients evaluated, 1033 (30.71\%) had UTIs [4]. The included studies provided different estimates of prevalence, which ranged from 16.0 to $75.0 \%$, and the pooled prevalence of UTIs was 38\% (95\% CI, 29-47\%; p < 0.01). Of note, RTx recipients followed for 1-2 years had significantly higher prevalence than those followed for 2-5 years (34 vs. $43 \%$ ).

\section{Definitions}

All UTIs can be classified into one of the four following categories:

(1) Asymptomatic bacteriuria $(\mathrm{AB})$, defined as isolation of bacterial strain in quantitative counts $\geq 105 \mathrm{CFU}$ in a clean-catch voided urine specimen in the absence of any symptoms of lower or upper UTI or $<10^{5} \mathrm{CFU}$ in patients treated with antibiotics or $\geq 10^{3} \mathrm{CFU}$ in a single catheterized urine specimen, irrespective of the presence of leukocyturia.

(2) Lower UTI, which is the presence of bacteriuria and clinical manifestations of dysuria, frequency or urinary urgency and fever $<38^{\circ} \mathrm{C}$ in the absence of acute graft pyelonephritis (AGPN) criteria.

(3) Upper UTI (AGPN), defined by the presence of significant bacteriuria, fever $>38^{\circ} \mathrm{C}$ and/ or graft pain and/or acute graft function impairment. 
(4) Urosepsis-life-threatening organ dysfunction caused by a dysregulated host response to the upper UTI.

Recurrent infections are defined as 3 or more episodes of symptomatic UTIs over a 12-month period or 2 episodes in the previous 6 months and can be divided into:

(1) Relapses: defined as the isolation of the same microorganism that caused the preceding infection in a urine culture obtained $\geq 2$ weeks after finishing the previous treatment. The isolation of the same microorganism that caused the preceding infection in a urine culture obtained $<2$ weeks after finishing the previous treatment should be considered a treatment failure.

(2) Reinfections: defined by a new episode of UTI with the isolation of an agent other than the one that caused the previous infection.

Definitions of multidrug-resistant (MDR) bacterial infections:

(1) Criteria for multidrug-resistant (MDR) bacteria: non-susceptible to $\geq 1$ agent in $\geq 3$ antimicrobial categories or methicillin resistance in the case of $S$. aureus.

(2) Criteria for extensively drug-resistant (XDR) bacteria: non-susceptible to $\geq 1$ agent in all but $\leq 2$ categories (i.e. bacterial isolates remain susceptible to only one or two categories).

(3) Criteria for pan drug-resistant (PDR) bacteria: non-susceptible to all the antimicrobials.

(4) Heteroresistance is defined as the presence of mixed populations of drug-resistant and drug-sensitive cells in a single clinical specimen.

\section{Predisposing factors for UTIs after RTx}

Many factors are believed to contribute to the high incidence of UTI in RTx recipients. Some exist prior to transplant, including female gender, diabetes mellitus and underlying urinary tract abnormalities. Peri-transplant factors are often related to instrumentation of the urinary tract, including ureteral stenting and prolonged urinary catheterization. Additional risk factors contributing to UTI post-transplant include immunosuppression and graft dysfunction or rejection. It is noteworthy that so far no direct association has been found between the risk of UTI and dose or type of maintenance immunosuppression. It is the net state of immunosuppression that impairs host defense capability against infections in general. Various authors have suggested different potential UTI risk factors, and their findings are not always consistent. The potential pre-, peri- and post-transplant risk factors for UTI in RTx recipients are shown in Table 1.

Of note, significant urine flow impairment, both existing pre-transplant or appearing posttransplant, seems to be of major importance. The bladder outlet obstruction, particularly in 


\begin{tabular}{lll}
\hline Pre-transplant & Peri-transplant & Post-transplant \\
\hline Urine flow impairment & Ureteral stents & Urine flow impairment \\
Female gender & Bladder instrumentation & - Vesicoureteral reflux (VUR) \\
Diabetes & Deceased-donor grafts & Strictures at the uretero-vesical junction \\
Urinary tract anomalies & Double kidney transplants & $\bullet \quad$ Benign prostate hyperplasia \\
Glomerulonephritis & & Immunosuppression \\
& & Acute rejection \\
& & Reduced graft function \\
\hline
\end{tabular}

Table 1. Risk factors for UTI in renal transplant recipients.

males, may not be appreciated until after the transplant, leading to prolonged instrumentation and an increased risk of UTI. The likelihood of AGPN development is 20-fold higher in patients with vesicoureteral reflux (VUR) or strictures at the uretero-vesical junction or benign prostate hyperplasia (BPH). Active reflux has long been reported as being significantly associated with poor graft outcome [5]. In a study by Dupont et al., VUR was found in almost half of RTx patients with recurrent UTIs, and patients with VUR were more prone to develop renal scarring than those without VUR [6]. On the other hand, in a recent study by Margreiter et al., $40 \%$ of 646 consecutive RTx recipients were diagnosed with VUR by voiding cystourethrography, and VUR did not affect the occurrence of UTIs. Simple UTI was diagnosed in $24.7 \%$ of patients with VUR and $27.2 \%$ of patients without VUR ( $p=0.78)$. Recurrent UTIs were noted in $4.2 \%$ (with VUR) versus $3.9 \%$ (without VUR) of the enrolled patients $(p=0.67)$. However, the authors did not analyze the incidence of UTI according to VUR grade [7]. In a retrospective cohort of 23,622 adult male primary RTx recipients, also benign prostate hyperplasia was independently associated with recurrent UTI [8]. Considering the significant influence of urinary flow abnormalities on the likelihood of AGPN development, we would strongly recommend examination for VUR or urine flow obstruction even at the first AGPN episode.

\section{Impact on renal allograft function}

The reports on the influence of UTIs on long-term kidney allograft function are inconsistent. The true impact of the whole spectrum of clinical manifestations of UTIs, on patient and graft outcome, so far has not been established. The general assumption is that asymptomatic bacteriuria $(\mathrm{AB})$ is benign, as opposed to acute graft pyelonephritis or urosepsis. Still, the paucity of symptoms might be attributable to immunosuppression with actual ongoing inflammation of unrecognized significance. In one small study, kidney transplant patients with asymptomatic bacteriuria had elevated urine IL-8 level; and the authors hypothesized that this phenomenon may reflect an impaired immune response to bacterial infection and occult inflammatory process in the urinary tract [9]. Pellé et al. showed that acute graft pyelonephritis (AGPN) was 
an independent risk factor for the decline in renal function in a group of 172 RTx recipients [10]. Also a more recent study analyzing the effects of recurrent UTIs on graft and patient outcomes, in a population of 2469 RTx recipients, showed both poorer graft and patient survival in patients with a history of $\geq 3$ UTIs in any 12-month period or $\geq 2$ UTIs in any 6-month period, irrespective of the causative organism [11]. However other reports did not confirm this relationship. Not only asymptomatic bacteriuria but also AGPN did not affect long-term renal graft function prognosis [12-15]. However, even if UTIs do not influence graft survival directly, they can pose a significant risk indirectly by leading to bacteraemia, acute rejection or cytomegalovirus (CMV) infection.

\section{Aetiology and timing of infections}

UTI after kidney transplantation is most often caused by Gram-negative organisms (around 50-90\%), with Escherichia coli as the most frequently isolated microorganism in urine cultures, similarly to general population. However, aetiology differs between the early and late periods after RTx [16]. Enterococcus species has emerged as an important pathogen and now
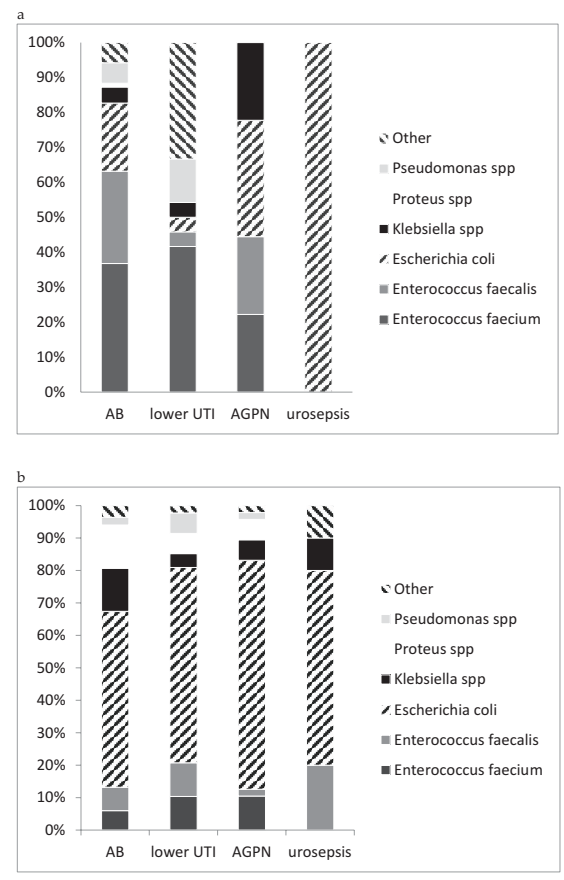

Figure 1. Proportion of different causative agents according to the type of UTI (a) during the first month post-transplant and (b) during 2-12 months post-transplant [16]. 
accounts for up to $30 \%$ of UTIs, especially in the first post-transplant month. In a study by Alangaden et al. Enterococcus spp. accounted for 33\% of UTIs, but authors failed to identify any specific risk factor associated with the predominance of this uropathogen [17]. Also in a study by Bonkat et al., Enterococcus spp. were bacteria most commonly responsible for microbial ureteral stent colonization in RTx recipients. The authors found two possible explanations for this phenomenon. Enterococcus spp. possess biofilm formation properties on various kinds of indwelling medical devices; and routine urine cultures often fail to identify biofilm forming Gram-positive pathogens, unlike the sonication technique used in that study to dislodge adherent microorganisms [18]. Another possible explanation of a high number of Enterococcus spp. infections is the routine use of cephalosporins in perioperative prophylaxis. This antibiotic acts against Gram-negative Bacilli, therefore it promotes selection of Enterococcus spp.

Beginning from the second month, Escherichia coli is the most frequently isolated causative agent, followed by Proteus spp., Klebsiella spp., Enterobacter spp. and Pseudomonas spp. (Figure 1).

\section{Multidrug-resistant bacteria}

With the widespread use of antibiotics, including the routine use of antimicrobial prophylaxis in RTx recipients, the prevalence of multidrug resistance (MDR) among uropathogenic bacteria is increasing, irrespectively of region and country. The most widely accepted definition of MDR includes lack of susceptibility to one or more agents in three or more antimicrobial categories active against the isolated bacteria. Of note, also extensively drug-resistant (XDR) and pan drug-resistant (PDR) strains have been identified.

In patients receiving trimethoprim-sulfamethoxazole prophylaxis, over $60 \%$ of UTIs have been reported as caused by trimethoprim-sulfamethoxazole-resistant organisms [19]. The treatment of $A B$ has also been associated with antimicrobial resistance. In a study of patients with asymptomatic E. coli or E. faecalis bacteriuria, treatment led to selection of resistant organisms in almost $80 \%$ of treated cases [20]. The emergence of ESBL-producing, or carbapenemaseproducing, organism pathogens has been the most important threat in nosocomial infections in recent years [21]. Although antibiotic resistance has been a concern since the introduction of penicillin, the past two decades have seen a marked increase in resistance, especially related to beta-lactams. Resistance in Gram-negative pathogens continues to increase, with multidrug resistance in the Enterobacteriaceae becoming one of the most important crises faced by the medical community. A major contributing factor is the acquisition of large plasmids that can encode resistance factors for multiple drug classes. As seen from the recent literature, organisms such as E. coli and the Klebsiella are acquiring more diverse integrons and transposons that are included in a multiplicity of transferable plasmids capable of encoding every class of beta-lactamase.

It seems that immunosuppression may influence the resistance of enterococcal spp. to $\beta$-lactambased antibiotics by affecting the expression of the penicillin-binding proteins (PBPs). In enterococcal strains isolated from RTx patients, the expression of the PBP5 gene was higher than in 
commensal strains. As cyclosporine seemed to promote higher expression of PBP5 than tacrolimus, $\beta$-lactam antibiotics may be more effective when tacrolimus-based immunosuppression protocols are implemented [22].

In a recently published study analyzing recurrent UTIs in a cohort of 2469 RTx recipients, the authors found pronounced differences in antimicrobial resistance patterns between nonrecurrent and recurrent UTIs [11]. Isolates from the cases of recurrent UTIs were more likely to be resistant to first- and third-generation cephalosporins, trimethoprim-sulfamethoxazole, nitrofurantoin and fluoroquinolones, to extended-spectrum b-lactams and aminoglycosides.

In a retrospective case series by Winters et al., $85 \%$ of solid-organ transplant recipients diagnosed with infection due to ESBL-producing bacteria received inadequate empiric therapy [23]. This means that all RTx recipients with a history of UTI due to ESBL-producing Gramnegative pathogens, presenting with symptoms of a new UTI, should receive an empiric therapy with a carbapenem until a urine culture result with susceptibility profile is available.

\section{Diagnosis}

UTIs in RTx recipients may either be asymptomatic or have an atypical clinical presentation. Therefore the diagnosis based solely on clinical grounds may be of questionable accuracy. What is more, every symptomatic, either lower or upper UTI in any transplant recipient, is considered complicated: as it is associated with structural and functional abnormalities of the genitourinary tract and immunocompromised status that increases the risk for acquiring an infection or of failing therapy. For this reason urine cultures should be obtained in every single case, in order to base therapy upon susceptibility pattern determinations.

\section{Asymptomatic bacteriuria}

Asymptomatic bacteriuria is a frequent finding in kidney allograft recipients, with almost $40 \%$ incidence [16]. So far there are no evidence-based recommendations for screening and treatment of $\mathrm{AB}$ in renal transplant recipients, because sufficient data is lacking [24]. The American Society of Transplantation Infectious Diseases Guidelines recommend limiting screening to the first post-transplant month, but these recommendations are mostly expert opinion [25]. Fiorante et al. showed that the incidence of AGPN was significantly higher in patients with a history of multiple episodes of $\mathrm{AB}$ than in patients without, despite or due to the provided antibiotic treatment [26].

Patients with no episodes of $\mathrm{AB}$ seem to develop significantly fewer symptomatic infections than patients with a history of recurrent $A B$. As reinfections seem to outnumber relapses and only a very few episodes of symptomatic UTIs are preceded by AB with the same causative agent in patients with a history of recurrent $A B$, it seems that $A B$ is more of a marker of increased susceptibility to infections, not a direct risk factor [16]. This is in agreement with the findings from a non-transplant population of young women, where the treatment of $A B$ in patients affected by recurrent UTI was associated with a higher rate of symptomatic UTI [27]. 
The authors hypothesized that this phenomenon resulted from ecological effects of antibacterial agents on the human microflora. However, Rice et al. found an association between $A B$ progression to systemic infection with acute kidney allograft injury and a unique pattern of adherence factors that is P fimbriae but not Dr. fimbriae expression [28]. So, AB might be an actual risk factor for symptomatic UTIs depending on the virulence of uropathogens.

A number of studies attempted to elucidate if the treatment of AB in RTx patients is in fact helpful or harmful in preventing symptomatic infections [19, 20]. One retrospective observational study included a total of 112 patients with $\mathrm{AB}$. The decision as to whether, or not, to treat $\mathrm{AB}$ was made by the attending physician. The primary outcome, defined as hospitalization for symptomatic UTI or a $25 \%$ decline in the eGFR, occurred more frequently among patients treated with antibiotics. However, the authors called attention to the fact that those treated patients may have initially been at higher risk for adverse outcomes, thus masking the benefit of the treatment [19]. Another retrospective study included 77 RTx recipients who developed $334 \mathrm{AB}$ episodes later than 1-month post transplantation. $\mathrm{AB}$ episodes were classified into four groups depending on the presence of pyuria and grade of bacteriuria. Spontaneous bacterial clearance occurred in 59\% of untreated episodes. The resolution of bacteriuria was not more frequent in treated, as compared to untreated, episodes. However, antibiotic treatment in patients with high-grade bacteriuria and concurrent pyuria resulted more frequently in negative control cultures than untreated episodes. The authors concluded that a watch-and-wait strategy for bacteriuria in the absence of pyuria might be safe in the RTx population [20]. In 2016, the results of a randomized controlled study were published. Systematic screening and treatment of $\mathrm{AB}$ beyond the second month after transplantation provided no apparent benefit among KT recipients when the occurrence of acute pyelonephritis at 24-month follow-up was considered. The treatment also did not affect the secondary outcomes, which included lower UTI, acute rejection, Clostridium difficile infection, colonization or infection by multidrug-resistant bacteria, graft function and all-cause mortality [29].

\section{Treatment}

Selection of initial empiric treatment should be based on local epidemiological data and the patient's history of resistant organisms. Once susceptibility data are available, the initial therapy should be deescalated, so that the most narrow-spectrum antibiotic is used to complete the course of therapy. Care should be taken to avoid treating asymptomatic patients, in order to reduce the possibility of infection with MDR pathogens.

Lower UTIs require minimum 7-day therapy with an effective agent while upper UTIs at least 2-3 weeks. The resolution of infection should be demonstrated before the cessation of treatment. Stents or catheters may be covered with bacterial biofilm, so their removal is generally required for resolution of UTI. For empirical treatment of suspected bacterial infections in RTx patients, the selection of antimicrobial agents should be based on local epidemiological data and on the patient's history of colonization or infection with antibiotic-resistant organisms. 
There is no evidence to support the use of combination antibiotic therapy for the treatment of ESBL, but in haemodynamically unstable or critically ill patients, adding an aminoglycoside to carbapenem seems a reasonable strategy. Amoxicillin-clavulanate and fosfomycin showed a clinical efficacy of 84 and $93 \%$, respectively, in the treatment of cystitis caused by ESBL-producing E. coli but only when the isolate showed susceptibility to those drugs [30]. Other options in the case of proven susceptibility include tigecycline, cotrimoxazole, quinolones and nitrofurantoin.

The combination antibiotic therapy is a standard of care in carbapenemase-producing Enterobacteriaceae infections [31, 32]. Colistin is the most active agent against these strains and should be considered the basis of treatment in most patients [33]. The options for the use of combination antibiotic therapy include aminoglycosides, fosfomycin or even high-dose carbapenems [31, 32, 34]. Tigecycline could represent an optimal choice for patients with co-infection with additional MDR pathogens [e.g. vancomycin-resistant Enterococci (VRE) or methicillin-resistant Staphylococcus aureus (MRSA)].

In the case of severe infection with sepsis, the option of reduction/discontinuation of immunosuppression together with surgical/urological intervention should also be considered.

In severe upper UTIs and/or recurrent infections, imaging should always be obtained to rule out structural causes or persistent foci of infection. Ultrasound may confirm the presence of hydronephrosis. When there are no visible structural abnormalities on ultrasound, it may be necessary to perform fluoroscopic voiding cystourethrogram to diagnose severe vesicoureteral reflux (VUR), computed tomography urography to visualize the cause of urine flow obstruction or uroflowmetry to recognize the problem with delayed bladder emptying. In elderly RTx recipients, the aforementioned functional abnormalities may be secondary to benign prostate hyperplasia (BPH). Since most patients undergoing dialysis are oliguric or anuric, urinary obstruction due to $\mathrm{BPH}$ and related lower urinary tract symptoms become evident after RTx and restoration of diuresis. As opposed to native kidneys, the transplanted kidney's ureter is shorter, and there is no valve at the vesicoureteral junction preventing backflow, so low-grade BPH may cause symptoms that would not be present in a non-RTx patient. Medical therapy of $\mathrm{BPH}$, both pharmacologic and surgical, such as transurethral resection of the prostate is safe and improves urinary flow and bladder emptying, to allow a significant and durable improvement of the kidney allograft function.

\section{Prevention and prophylaxis}

Appropriate attention should be given to the prevention of UTI with correction of structural abnormalities of the urinary tract in the potential RTx recipients prior to transplantation. Any type of voiding dysfunction should be considered and addressed.

In the immediate post-transplant period, vigilance for donor-transmitted infection is important, together with routine perioperative antibiotic prophylaxis recommended by the hospital's epidemiologist, taking into account current antibiotic resistance of Gram-negative strains. In the 
case of a positive donor's or organ preservation fluid cultures, the antibiotic should be chosen according to susceptibility profiles. The use of indwelling urethral catheters and ureteral stents should be minimized.

Patients should be instructed to drink a lot of fluids and urinate frequently, without waiting for the urge to urinate.

There is no consensus regarding the optimal strategy and duration of recurrent UTI prophylaxis, so the decision to give it, or not, depends on the experience of the treating physician. Traditionally trimethoprim/sulfamethoxazole prophylaxis has been used as the prevention of both asymptomatic bacteriuria/UTI and Pneumocystis pneumonia after RTx. However, over the past few years, it has become less effective as uropathogens have become more resistant to this regimen. Of note, ESBL-producing E. coli are usually susceptible to nitrofurantoin, while most Klebsiella spp. strains are resistant to this antibiotic.

Several possibilities exist in an attempt to mitigate the damage caused by resistant pathogens. In the general population, there are ongoing attempts to use nonantibiotic strategies, such as cranberry products, D-mannose, probiotics, immunoactive prophylaxis with several types of vaccines, intravesical glycosaminoglycan replenishment therapy with the use of chondroitin sulfate and low molecular weight hyaluronic acid in the treatment and/or prevention of recurrent UTIs [35]. So far the use of all these products has not been extensively studied in RTx population, except for single-case reports on the use of cranberry products. Little information is also available about the usefulness of intestinal decolonization in RTx patients.

\section{Author details}

Justyna Gołębiewska* and Alicja Dębska-Ślizień

*Address all correspondence to: jgolebiewska@gumed.edu.pl

Department of Nephrology, Transplantology and Internal Medicine, Medical University of Gdańsk, Poland

\section{References}

[1] Veroux M, Giuffrida G, Corona D, Gagliano M, Scriffignano V, Vizcarra D, Tallarita T, Zerbo D, Virgilio C, Sciacca A, Cappello D, Stefani S, Veroux P. Infective complications in renal allograft recipients: Epidemiology and outcome. Transplantation Proceedings. 2008; 40:1873-1876

[2] Gołębiewska J, Dębska-Ślizień A, Komarnicka J, Samet A, Rutkowski B. Urinary tract infections in renal transplant recipients. Transplantation Proceedings. 2011;43:2985-2990

[3] Nampoory MR, Johny KV, Costandy JN, Nair MP, Said T, Homoud H, Al-Muzairai I, Samhan M, Al-Moussawi M. Infection related renal impairment: A major cause of acute allograft dysfunction. Experimental and Clinical Transplantation. 2003;1:60-64 
[4] Wu X, Dong Y, Liu Y, Li Y, Sun Y, Wang J, Wang S. The prevalence and predictive factors of urinary tract infection in patients undergoing renal transplantation: A meta-analysis. American Journal of Infection Control. 2016;44(11):1261-1268

[5] Mathew T, Kincaid-Smith P, Vikraman P. Risks of vesicoureteric reflux in the transplanted kidney. The New England Journal of Medicine. 1977;297:414-418

[6] Dupont PJ, Psimenou E, Lord R, Buscombe JR, Hilson AJ, Sweny P. Late recurrent urinary tract infections may produce renal allograft scarring even in the absence of symptoms or vesicoureteric reflux. Transplantation. 2007;84:351-355

[7] Margreiter M, Györi GP, Böhmig GA, Trubel S, Mühlbacher F, Steininger R. Value of routine voiding cystourethrography after renal transplantation. American Journal of Transplantation. 2013;13:130-135

[8] Hurst FP, Neff RT, Falta EM, Jindal RM, Lentine KL, Swanson JS, Agodoa LY, Abbott KC. Incidence, predictors, and associated outcomes of prostatism after kidney transplantation. Clinical Journal of the American Society of Nephrology. 2009;4:329-336

[9] Ciszek M, Pączek L, Bartłomiejczyk I, Mucha K. Urine cytokines profile in renal transplant patients with asymptomatic bacteriuria. Transplantation. 2006;81:1653-1657

[10] Pellé G, Vimont S, Levy PP, Hertig A, Ouali N, Chassin C, Arlet G, Rondeau E, Vandewalle A. Acute pyelonephritis represents a risk factor impairing long-term kidney graft function. American Journal of Transplantation. 2007;7:899-907

[11] Britt NS, Hagopian JC, Brennan DC, Pottebaum AA, Santos CAQ, Gharabagi A, Horwedel TA. Effects of recurrent urinary tract infections on graft and patient outcomes after kidney transplantation. Nephrology, Dialysis, Transplantation. 2017;32:1758-1766

[12] Fiorante S, Fernandez-Ruiz M, Lopez-Medrano F, Lizasoain M, Lalueza A, Morales JM, San-Juan R, Andrés A, Otero JR, Aguado JM. Acute graft pyelonephritis in renal transplant recipients: Incidence, risk factors and long-term outcome. Nephrology, Dialysis, Transplantation. 2011;26:1065-1073

[13] Giral M, Pascuariello G, Karam G, Hourmant M, Cantarovich D, Dantal J, Blancho G, Coupel S, Josien R, Daguin P, Méchineau S, Soulillou JP. Acute graft pyelonephritis and long-term kidney allograft outcome. Kidney International. 2002;61:1880-1886

[14] Kamath NS, John GT, Neelakantan N, Kirubakaran MG, Jacob CK. Acute graft pyelonephritis following renal transplantation. Transplant Infectious Disease. 2006;8:140-147

[15] Singh R, Geerlings SE, Peters-Sengers H, Idu MM, Hodiamont CJ, Ten Berge IJ, Bemelman FJ. Incidence, risk factors, and the impact of allograft pyelonephritis on renal allograft function. Transplant Infectious Disease. 2016;18:647-660

[16] Gołębiewska JE, Dębska-Ślizień A, Rutkowski B. Treated asymptomatic bacteriuria during first year after renal transplantation. Transplant Infectious Disease. 2014;16:605-615

[17] Alangaden GJ, Thyagarajan R, Gruber SA, Morawski K, Garnick J, El-Amm JM, West MS, Sillix DH, Chandrasekar PH, Haririan A. Infectious complications after kidney transplantation: Current epidemiology and associated risk factors. Clinical Transplantation. 2006;20:401 
[18] Bonkat G, Rieken M, Siegel FP, Frei R, Steiger J, Gröschl I, Gasser TC, Dell-Kuster S, Rosenthal R, Gürke L, Wyler S, Bachmann A, Widmer AF. Microbial ureteral stent colonization in renal transplant recipients: Frequency and influence on the short-time functional outcome. Transplant Infectious Disease. 2012;14:57

[19] Green H, Rahamimov R, Gafter U, Leibovitci L, Paul M. Antibiotic prophylaxis for urinary tract infections in renal transplant recipients: A systematic review and meta-analysis. Transplant Infectious Disease. 2011;13:441-447

[20] El Amari EB, Hadaya K, Buhler L, et al. Outcome of treated and untreated asymptomatic bacteriuria in renal transplant recipients. Nephrology, Dialysis, Transplantation. 2011;26:4109-4114

[21] Philippon A, Arlet G, Lagrange PH. Origin and impact of plasmid-mediated extendedspectrum beta-lactamases. European Journal of Clinical Microbiology \& Infectious Diseases. 1994;13(Suppl 1):S17

[22] Jarzembowski T, Daca A, Witkowski J, Rutkowski B, Gołębiewska J, Dębska-Ślizień A. Changes of PBP5 gene expression in enterococcal isolates from renal transplantation recipients. BioMed Research International. 2013;2013:687156

[23] Winters HA, Parbhoo RK, Schafer JJ, et al. Extended-spectrum beta-lactamase producing bacterial infections in adult solid organ transplant recipients. The Annals of Pharmacotherapy. 2011;45:309

[24] Nicolle LE, Bradley S, Colgan R, Rice CJ, Schaeffer A, Hooton TM. Infectious Diseases Society of America Guidelines for the diagnosis and treatment of asymptomatic bacteriuria in adults. Clinical Infectious Diseases. 2005;40(5):643-654

[25] Parasuraman R, Julian K, The AST Infectious Diseases Community of Practice. The American Society of Transplantation Infectious Diseases Guidelines 3rd edition. Urinary tract infections in solid organ transplantation. American Journal of Transplantation. 2013;13(Suppl 4):327-336

[26] Fiorante S, Lopez-Medrano F, Lizasoain M, et al. Systematic screening and treatment of asymptomatic bacteriuria in renal transplant recipients. Kidney International. 2010; 78(8):774-781

[27] Cai T, Mazzoli S, Mondaini N, et al. The role of asymptomatic bacteriuria in young women with recurrent urinary tract infections: To treat or not to treat? Clinical Infectious Diseases. 2012;55:771-777

[28] Rice JC, Peng T, Kuo Y-F, et al. Renal allograft injury is associated with urinary tract infection caused by Escherichia coli bearing adherence factors. American Journal of Transplantation. 2006;6:2375-2383

[29] Origüen J, López-Medrano F, Fernández-Ruiz M, et al. Should asymptomatic bacteriuria be systematically treated in kidney transplant recipients? Results from a randomized controlled trial. American Journal of Transplantation. 2016;16:2943-2953 
[30] Rodriguez-Bano J, Alcala JC, Cisneros JM, et al. Community infections caused by extendedspectrum beta-lactamase-producing Escherichia coli. Archives of Internal Medicine. 2008;168:1897-1902

[31] Clancy CJ, Chen L, Shields RK, et al. Epidemiology and molecular characterization of bacteremia due to carbapenem-resistant Klebsiella pneumoniae in transplant recipients. American Journal of Transplantation. 2013;13:2619-2633

[32] Bergamasco MD, Barroso Barbosa M, de Oliveira Garcia D, et al. Infection with Klebsiella pneumoniae carbapenemase (KPC)-producing K. pneumoniae in solid organ transplantation. Transplant Infectious Disease. 2012;14:198-205

[33] Gales AC, Jones RN, Sader HS. Contemporary activity of colistin and polymyxin B against a worldwide collection of Gram-negative pathogens: Results from the SENTRY antimicrobial surveillance program (2006-09). The Journal of Antimicrobial Chemotherapy. 2011;66:2070-2074

[34] Taglietti F, Di Bella S, Galati V, Topino S, Iappelli M, Petrosillo N. Carbapenemase-producing Klebsiella pneumoniae-related mortality among solid organ-transplanted patients: Do we know enough? Transplant Infectious Disease. 2013;15:E164-E165

[35] Costantini E, Giannitsas K, Illiano E. The role of nonantibiotic treatment of communityacquired urinary tract infections. Current Opinion in Urology. 2017;27:120-126 
